\title{
Branes, boundary CFT and non-commutativity
}

\author{
Andreas Recknagel * \\ Department of Mathematics, King's College London \\ Strand, London WC2R 2LS, U.K. \\ E-mail: 'anderl@mth.kcl.ac.uki
}

ABstract: Branes have a world-sheet description in terms of boundary conformal field theory, which allows to characterize possible branes for a given closed string background as possible conformal boundary conditions for a CFT on the plane. The world-sheet approach accommodates phenomena which seem to reach beyond the classical target space picture of branes and point towards a non-commutative texture of branes. We discuss the appearance of non-commutative geometry within brane physics for the example of branes in $\mathrm{SU}(2)$. In some respects, this situation is more complicated than the widely discussed case of flat branes with constant B-field, but it still offers an approximate classical description, where brane world-volumes are spherical or point-like conjugacy classes of $\mathrm{SU}(2)$. The 'traditional approach to non-commutativity' is based on a computation of the effective action of low-lying brane excitations, which generically leads to Lagrangians that are 'geometrical' only in an appropriate non-commutative sense. Concentrating on the infinite level limit of the SU(2) theory, one finds a Yang-Mills-Higgs action on a fuzzy two-sphere (the truncation quantization of a sphere), complemented by a Chern-Simons term. Studying solutions to the effective equations of motion with the help of techniques from conformal perturbation theory, one is led to interpreting spherical branes as bound states of point-like branes. We also discuss how a more direct, though somewhat speculative, link between branes and non-commutative geometry can be established by defining an algebra of 'functions on the brane world-volume' in terms of the boundary CFT data.

\section{Introduction}

In the past year, there has been a lot of excitement and a lot of activity concerned with the role of non-commutative geometry in string theory; see e.g. Barbon's talk for more details and for references. That non-commutative, or at least some non-classical geometry is important for string theory has of course long been suspected, simply because string theory in particular aims at quantising gravity. Even if one does not want to use arguments about the metric becoming a quantum operator, and can conclude [1] from Heisenberg's uncertainty relations and the existence of black holes that there are uncertainty relations for the coordinates themselves, a strong indication of non-commutative geometry.

\footnotetext{
*Talk based on joint work with A.Yu. Alekseev, V. Schomerus and D. Roggenkamp, and with J. Fröhlich and O. Grandjean
}

Having this kind of reasoning in mind, the noncommutative effects encountered in brane physics are indeed somewhat unexpected: Gravity is not involved, and moreover the new phenomena on branes arise at energies much lower than the string scale.

One of the main points we would like to make here is that still, from the world-sheet point of view, the appearance of non-commutative geometry in low-energy brane physics is no more surprising that the appearance of classical geometry: Both appear on the same footing. More to the point, neither non-commutative geometry nor classical geometry are needed as an ingredient of the world-sheet theory, they merely provide more or less convenient interpretations added afterwards.

To support this perspective, we present in section 5 a world-sheet computation of the low-energy ef- 
fective action of open string attached to a brane in $\mathrm{SU}(2)$. Geometry is never needed in the process, but on reason for choosing this example is of course that there is a competing classical target picture of the situation. In particular, one can observe how open string effects seem to 'quantise' a classical brane world-volume, and noncommutativity can be seen to be ultimately due to non-locality of open string vertex operators.

Although the classical picture for brane in group manifolds is still rather simple, concrete results (like a LEEA) are difficult to obtain within the target space setting: Group manifolds are significantly less trivial than the flat backgrounds mainly discussed in the string literature in that are curved with non-vanishing Ricci tensor, and the string equation of motion $R_{i j} \sim H_{i k l} H_{j}{ }^{k l}$ implies that (to order $\alpha^{\prime}$ ) the target carries not only a non-vanishing, but indeed a non-constant B-field. Therefore, we expect branes in group manifolds to exhibit non-commutative effects at least as interesting as those for flat branes in constant B-fields. The CFT computations of section 5 will show that this is indeed the case.

In the next section, we briefly review some elements of the boundary CFT language needed for the world-sheet description of branes. We also collect some data on boundary conditions in SU(2) WZW models for later use. Section 3 review how non-commutativity appears in connection with flat branes in a B-field, mainly in order to display the guidelines towards and open string LEEA, be followed again later in the more involved SU(2) case. The classical picture of branes in group manifolds is sketched in Section 4, so as to prepare a contrasting background for the purely CFT manipulations in Section 5, which lead to a LEEA for brane in SU(2). This action can be recognised as a gauge theory (Yang-Mills and Chern-Simons) on a fuzzy sphere, in accordance with the qualitative discussion of Section 4. Investigation of solutions to the equations of motion derived from the LEEA displays interesting dynamics of these branes: In particular, spherical branes can be realised as boundary states of point-like branes.

In spite of the generality and rigour of CFT calculations, one cannot deny the efficiency (and sometimes the beauty) of geometrical principles, in particular when effective actions are extracted from string theory. However, the existence of non-commutative brane world-volumes shows that classical geometry is too narrow a framework, and that one should widen the scope of geometric principles appropriately for application in string theory. The concluding section contains some rather speculative remarks on how to link the world-sheet description of branes to non-commutative geometry. In particular, we propose a procedure which assigns so-called spectral data - in Connes' framework, the defining data of a space - directly to a boundary CFT.

The results presented in section 5 are taken from

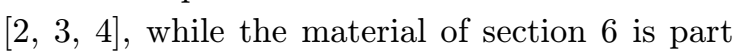

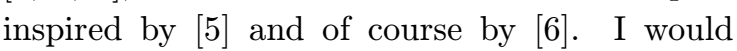
like to thank V. Schomerus, A.Yu. Alekseev, D. Roggenkamp and also J. Fröhlich and O. Grandjean for collaboration and numerous helpful and enjoyable discussions.

\section{Elements of boundary CFT}

Here we collect some basic facts about conformal field theory on the upper half-plane. Such boundary conformal field theories provide the worldsheet description of D-branes in the general sense - they are nothing but conformal boundary conditions for a given closed string background.

To such a closed string background there corresponds a 'bulk' CFT on the plane, with symmetry algebra $\mathcal{W} \times \mathcal{W}$ and a state space, whose decomposition into irreducibles of the symmetry algebra reads $\mathcal{H}^{\text {bulk }}=\bigoplus_{i, j} \mathcal{H}_{i} \otimes \mathcal{H}_{j}$. There is a one-to-one correspondence between $\mathcal{H}^{\text {bulk }}$ and the space of conformal bulk fields $\left(\varphi_{i j}(z, \bar{z})\right.$ and descendants). The construction of a boundary CFT for a given bulk CFT amounts to restricting the CFT to the upper half-plane in a consistent way, keeping the local structure (the OPE of bulk fields) in the interior.

The defining data of a conformal boundary condition can be split into two parts: First, linear conditions ('gluing conditions') that fix the symmetry of the boundary CFT. Secondly, choosing the values of certain coefficients in a consistent way. 
Choice of gluing conditions includes specification of a sub-W-algebra $\mathcal{W}_{\text {op }} \subset \mathcal{W}$ which contains the Virasoro algebra, $\operatorname{Vir} \subset \mathcal{W}_{\text {op }}$, and of a $W$ algebra automorphism $\Omega: \mathcal{W}_{\text {op }} \longrightarrow \mathcal{W}_{\text {op }}$ that acts trivially on the elements of Vir. Then we demand that

$$
W(z)=\Omega \bar{W}(\bar{z})
$$

for all $W \in \mathcal{W}_{\text {op }}$ and for $z=\bar{z}$, i.e. along the boundary of the upper half-plane $(z=x+i y$ with $y>0)$. If this conditions links the generators of left- and right-moving sub-symmetry algebras $\mathcal{W}_{\text {op }}$, one can show that the boundary CFT enjoys covariance under one copy of $\mathcal{W}_{\text {op }}$. The specific automorphism $\Omega$ e.g. enters the Ward identities that hold in the theory, see e.g $\left[\begin{array}{l}{[\bar{i}} \\ 1\end{array}\right]$.

Let us look at two simple examples and give gluing conditions for a system of $D$ free bosons and for non-abelian WZW models. In the first case, we have $\mathcal{W}=\mathrm{U}(1)^{D}$ with generators $J^{\mu}=\partial X^{\mu}$ (analogously for the right-movers). The usual gluing conditions are $J^{\mu}(z)=\bar{J}^{\mu}(\bar{z})$ (Neumann conditions) and $J^{\mu}(z)=-\bar{J}^{\mu}(\bar{z})$ (Dirichlet conditions). The first choice $\Omega=$ id is available in every CFT, even though its interpretation depends on the specific model, as we will see. For a non-abelian WZW model, where $\mathcal{W}$ is generated by currents $J^{a}(z)$, there are a number of natural gluing conditions,

$$
J^{a}=\bar{J}^{a} \quad \text { or } \quad J^{a}=\operatorname{Ad}_{h} \bar{J}^{a} \quad \text { or } \quad J^{a}=\Omega^{(o)} \bar{J}^{a}
$$

for some outer automorphism of the underlying Lie group (in case such automorphisms exist). We will discuss these gluing conditions in more detail in Section 4.

The second kind of data to fix when defining a boundary condition are certain (OPE) coefficients. This has to be done in such a way that various non-linear sewing relations are satisfied

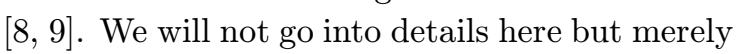
note that the simplest of these coefficients are those in the 1-point functions of primary bulk fields,

$$
\left\langle\varphi_{i j}(z \bar{z})\right\rangle_{\alpha}=\frac{A_{i j}^{\alpha}}{|z-\bar{z}|^{2 h_{i}}} .
$$

In contrast to CFTs on the plane, this expectation value need not vanish for $\varphi_{i j} \neq \mathbf{1}$, simply because translation invariance orthogonal to the boundary is broken. Rather, there may be various independent sets (labelled by $\alpha$ ) of coefficients $A_{i j}^{\alpha}$ which satisfy all sewing constraints. Each such solution specifies a conformal boundary condition, or a brane. In principle, once the 1-point functions are known one can compute arbitrary correlation functions of bulk fields in the presence of the boundary conditions (using the bulk OPE). One can further determine the partition function $Z_{\alpha}(q)$ of the boundary CFT [isi]. Within string theory, the $A_{i j}^{\alpha}$ specify tension and charges of the brane $\alpha$.

Let us describe a consistent set of $A_{i j}^{\alpha}$ for the $\mathrm{SU}(2)_{\mathrm{k}}$ WZW model with diagonal bulk partition function (i.e. only $i=j$ occurs) and for the 'standard gluing condition' $\Omega=$ id. Then one can choose [iإ]

$$
A_{i}^{\alpha}=\frac{S_{\alpha i}}{\sqrt{S_{0 i}}}
$$

where $S$ denotes the modular $S$-matrix for the chiral characters. In particular, the labels $\alpha$ for different boundary conditions (branes) run over the same set as the labels $i$ of the bulk primaries, namely $\alpha=0, \frac{1}{2}, \ldots, \frac{\mathrm{k}}{2}$; the ' 0 ' in (2. $\left.2.3 \mathrm{i}\right)$ is for the vacuum module. This kind of boundary conditions exists for a large class of rational CFTs, it is often called 'Cardy-type boundary condition'. Brane partition functions follow from $(2.2 .3)$ along the lines of [ip]; one obtains

$$
Z_{\alpha}(q) \equiv \operatorname{tr}_{\mathcal{H}_{\alpha}} q^{L_{0}-\frac{c}{24}}=\sum_{l} N_{\alpha \alpha}^{l} \chi_{l}(q)
$$

the $N_{i j}^{k}$ denote the $\mathrm{SU}(2)_{\mathrm{k}}$ fusion rules, and the summation runs over $l=0,1, \ldots, \min (2 \alpha, \mathrm{k}-$ $2 \alpha)$. In the middle of eq. (2.4), the state space $\mathcal{H}_{\alpha}$ of the boundary CFT with boundary condition $\alpha$ appears; its states are in one-to-one correspondence to the boundary fields of the theory which will play an important role later on.

For completeness, let us also give the partition function for the free boson with Neumann boundary conditions:

$$
Z_{N}(q)=\int^{\oplus} d k \frac{q^{k^{2} / 2}}{\eta(q)} ;
$$

$k$ labels the momentum of an open string vertex operator. 
Boundary fields are additional operators whose existence is made plausible by the fact that bulk field correlators usually have singularities when the fields approach the boundary, cf. (2.21). Boundary fields $\psi_{k}(x)$ falls into irreducible representations of the symmetry algebra $\mathcal{W}_{\text {op }}$. Just like chiral vertex operators, they have an operator product expansion $\left(x_{1}>x_{2}\right)$

$$
\sum_{m}\left(x_{1}\right) \psi_{l}\left(x_{2}\right)=
$$

and the coefficients are to be fixed in such a way as to satisfy sewing relation. As an important feature, let us stress that boundary fields in general are confined to the boundary of the worldsheet and cannot be moved into the interior. In particular, there is no unique way to interchange the two operators on the lhs of (2.51), thus the coefficients depend on the ordering of the arguments.

For free bosons as above, the ordinary OPE of open string vertex operators is recovered. The $\mathrm{SU}(2)$ with a 'Cardy type' boundary condition labelled by $\alpha$ contains $\mathrm{SU}(2)$-multiplets associated to primary boundary fields, namely $\psi_{j}^{J}(x)$ with $J=0,1, \ldots, \min (2 \alpha, \mathrm{k}-2 \alpha)$ and $j=-J, \ldots, J$. In this case, and indeed for Cardy type boundary conditions in any rational CFT, the coefficients in the boundary OPE above are known: They can be expressed in terms of the fusing matrix

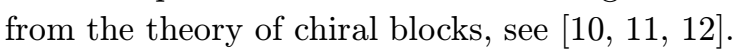
For the SU(2)-case, we have

$$
C_{(I, i)(J, j)(K, k)}^{\alpha}=\left[\begin{array}{lll}
I & J & K \\
i & j & k
\end{array}\right]\left\{\begin{array}{lll}
I & J & K \\
\alpha & \alpha & \alpha
\end{array}\right\}_{q}
$$

where th first symbol on the rhs is a ClebschGordan coefficient of the group SU(2), while the second is a $6 J$ symbol of the quantum group algebra $U_{q}(\operatorname{su}(2))$ with $q=e^{\frac{2 \pi i}{\mathrm{k}+2}}$. For our purposes, only the limit $\mathrm{k} \rightarrow \infty$ will be important. There, the structure constants above approach those of matrix multiplication in $\operatorname{Mat}(2 \alpha+1)$, see [i-in for details.

\section{Flat branes in a B-field}

Let us briefly recall the main features of how noncommutative effects are uncovered in the simple case of flat branes in $\mathbb{R}^{D}$ which carry a B-field; for more details, see e.g. $\left[1 \overline{1}, \overline{1}, \overline{1} \overline{4}_{1}^{\prime}\right.$. One can start from a $\sigma$-model description where the ordinary Polyakov action is supplemented by a boundary term

$$
S_{B} \sim F \int_{\partial \Sigma} d x X^{1} \partial X^{2}
$$

For simplicity, we display only two target dimensions with coordinates $X^{\mu}, \mu=1,2$. The constant $F$ is the strength of a (magnetic) B-field along these directions. Upon variation, the $\sigma$ model action in particular yields gluing conditions along the boundary $\partial \Sigma$ :

$$
\partial_{y} \vec{X}=-B \partial_{x} \vec{X} \text { with } B=\left(\begin{array}{cc}
0 & F \\
-F & 0
\end{array}\right)
$$

Of course, ordinary Neumann conditions are recovered when the B-field is switched off. For later convenience, let us rewrite $(\overline{3} .1)$ in the form

$$
\partial \vec{X}=\widetilde{\Theta} \bar{\partial} \vec{X} \text { with } \widetilde{\Theta}=\frac{1-B}{1+B} .
$$

If one wants to compute the low-energy effective action for branes in a B-field, the main ingredients one needs from CFT are the spectrum of open string vertex operators (boundary fields) allowed by the boundary condition, and their interactions. In the present case of an uncompactified target, one finds that the boundary field content is not influenced by the magnetic field, while the boundary OPE does depend on it. This can be seen e.g. by computing $\left\langle X\left(x_{1}\right) X\left(x_{2}\right)\right\rangle$ from the gluing conditions $(\overrightarrow{3} \cdot \overrightarrow{2})$. For primary vertex operators $V[\vec{k}]=\exp \{i \vec{k} \cdot \vec{X}\}$, this yields the OPE

$$
\begin{aligned}
& e^{i \vec{k}_{1} \cdot \vec{X}}\left(x_{1}\right) e^{i \vec{k}_{2} \cdot \vec{X}}\left(x_{2}\right)= \\
& \left|x_{1}-x_{2}\right|^{\alpha^{\prime} \vec{k}_{1}^{\mathrm{t}} G \vec{k}_{2}} e^{i \pi \vec{k}_{1}^{\mathrm{t}} \Theta \vec{k}_{2}} e^{i\left(\vec{k}_{1}+\vec{k}_{2}\right) \cdot \vec{X}}\left(x_{2}\right)+\ldots
\end{aligned}
$$

where $G$ and $\Theta$ are proportional to the symmetric resp. anti-symmetric part of $\widetilde{\Theta}$. In the expansion (3.3), it is understood that vertex operators are ordered such that $x_{1}>x_{2}$ : This is crucial (for non-vanishing B-field) because of the anti-symmetry of $\Theta$. The massless effective fields showing up in the effective action are the 'parameters' $A_{\mu}$ in $\Psi=A_{\mu}(k): \partial X^{\mu} V[k]:$. The $n$th order (in $A_{\mu}$ ) contribution to $\mathcal{S}_{\text {eff }}$ is obtained from the CFT $n$-point function of the boundary field $\Psi$, after integrating out world-sheet moduli. 
In addition, it is essential to specify the regime of parameters where the effective action is to be evaluated: Taking $\alpha^{\prime} \rightarrow 0$ simply means that we only look at massless open string excitations, but for the following it is equally important to scale the other parameters appropriately, as was emphasised in [1 14 ']. This 'Seiberg-Witten limit' requires to keep the open string metric $G$ and the anti-commutativity parameter $\Theta$ fixed. Looking at $(13.3)$, we see that this implies that the $V[\vec{k}]$ OPE becomes topological in the sense that the $\left(x_{1}-x_{2}\right)$-dependence disappears, but the ordering of the vertex operators still affects the $\Theta$ dependent phase. In this specific limit one finds that the low-energy effective action of flat branes in a B-field is given by

$$
\mathcal{S}_{\mathrm{eff}}=\int_{\mathbb{R}^{2}} F * F
$$

(up to higher order terms in $A_{\mu}$ ) with

$$
F_{\mu \nu}=\partial_{\mu} A_{\nu}-\partial_{\nu} A_{\mu}+i A_{\mu} * A_{\nu}-i A_{\nu} * A_{\mu} .
$$

This result was first obtained in [1 15 - 5 ; the methods used there are based on target space considerations and do not easily generalise to other situations, but in particular they led to the insight that the Moyal product

$$
(f * g)(x)=\left.e^{-i \pi \Theta^{\mu \nu} \partial_{\mu}^{x} \partial_{\nu}^{y}} f(x) g(y)\right|_{x=y}
$$

enters the effective action. $\mathcal{S}_{\text {eff }}$ is well known to describe Maxwell theory on a non-commutative plane, and therefore one can suggest in hindsight that the classical world-volume of our 2dimensional brane has undergone a deformation quantisation through quantum effects in the presence of open strings and a B-field. If asked to give the 'world-sheet reason' for non-commutativity, we can point to the fact that the OPE of vertex operators depends on their ordering even in the simplifying limit considered above. In view of what was said before about non-locality of boundary fields, we expect that non-commutativity is virtually omnipresent in brane physics.

\section{D-branes in $\mathrm{SU}(2)$}

As was mentioned before, our special interest in branes in WZW models rests partially on the fact that these conformal field theories have a classical target space description, as $\sigma$-models on group manifolds. Thus we can contrast classical pictures to the results of an exact CFT analysis, which fortunately can be worked out in great detail for these theories.

Even though most of the following statements have an immediate generalisation to other groups, we will mainly stick to the case $G=\mathrm{SU}(2)$, which is not only distinguished by its simplicity, but also because this group manifold occurs as component of consistent superstring backgrounds like $\mathrm{AdS}_{3} \times S^{3} \times T^{4}, \mathrm{AdS}_{3} \times S^{3} \times S^{3} \times S^{1}$ or $\mathbb{R}^{5,1} \times$ $\mathbb{R}_{>0} \times S^{3}$. In the latter example, which provides the world-sheet description of Neveu-Schwarz 5branes [1 $\left.{ }_{1}^{1}{ }_{1}^{\prime}\right]$, the middle factor refers to a linear dilaton, i.e. a Liouville theory.

In order to construct conformal boundary conditions in the WZW target space, it is by far most convenient to start from the currents' gluing conditions of the world-sheet description and then insert the relations

$$
J(z)=-\mathrm{k}(\partial g) g^{-1} \quad, \quad \bar{J}(\bar{z})=\mathrm{k} g^{-1} \bar{\partial} g
$$

to the group-valued field $g: \Sigma \rightarrow G$.

Using this, it was shown in $\left[1 \overline{1}_{1}\right]$ - but see also

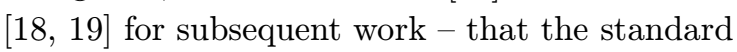
gluing conditions $J^{a}(z)=\bar{J}^{a}(\bar{z})$ correspond, in the classical limit, to the constraint that $g(\partial \Sigma)$ takes values in a conjugacy class of $G$.

For $\mathrm{SU}(2)$, we have two point-like conjugacy classes $\pm e$ (the central elements) and, the generic case, two-spheres within $S^{3} \simeq \mathrm{SU}(2)$, symmetric about the axis through $e$ and $-e$ and parameterised by an angle $\theta \in] 0, \pi[$.

Obviously, this orientation within the target is not preferred and one can indeed obtain the condition that $g(\partial \Sigma)$ lies in a rotated conjugacy class when one starts from the CFT gluing conditions $J^{a}(z)=\operatorname{Ad}_{h} \bar{J}^{a}(\bar{z})$ for some group element $h$ [1] $\overline{1} \bar{j}$. Within the CFT, such twists by inner automorphisms $\Omega=\Omega^{(i)}$ can also be realized as deformations by marginal boundary fields [ $[201]$, in accordance with the symmetries of the target space.

For more complicated groups, also outer automorphisms $\Omega=\Omega^{(o)}$ are available for insertion into the gluing conditions; they lead to twisted conjugacy classes of a different form. E.g., in the 
$\mathrm{SU}(3)$ case, the generic twisted conjugacy class has a classical world-volume of codimension 1 , instead of codimension 2 as for $\Omega=$ id.

All the boundary conditions mentioned so far are maximally symmetric in the sense that the full affine Lie algebra associated to $G$ acts in the boundary CFT. One should stress that already in these cases, the classical world-volumes have more structure than one would naively have expected from the simple "Neumann-like" appearance of standard gluing conditions; in particular, note that none of the above branes is "targetfilling". From the CFT point of view, it is conceivable that there are additional boundary conditions which preserve only the Virasoro algebra. To establish this and construct them is a difficult task because the resulting models are no longer rational. But if they exist, it should be a very interesting problem to find a geometrical target space interpretation for their world-volumes - in case there is one.

Returning to the basic case of the standard gluing conditions $J^{a}(z)=\bar{J}^{a}(\bar{z})$, the analysis that uncovers the role of conjugacy classes also shows that the generic branes carry a non-vanishing Bfield [17]. The gluing conditions can be cast into the form

$$
\left(g^{-1} \partial_{y} g\right)^{\|}=\frac{\operatorname{Ad}(g)+1}{\operatorname{Ad}(g)-1}\left(g^{-1} \partial_{x} g\right)^{\|}
$$

where $(\cdot)^{\|}$denotes projection along the tangent space of a conjugacy class. The interpretation of the quotient on the rhs as B-field follows from the usual correspondence between $\sqrt{\mathrm{k}} g^{-1} \partial g$ and the flat space coordinate $\partial X$, cf. also eq. (13.1). The associated 2-form $B$ is a particular potential for the NS 3-form field strength $H=d B$, within a neighbourhood of the conjugacy class. Later, we will mostly be concerned with the large level limit of the $\mathrm{SU}(2)$ theory. In appropriate coordinates the field strength decays with $1 / \mathrm{k}$ while the Bfield is level independent.

Let us add that a semi-classical extension of the above analysis, e.g. using path integral methods [1] 1 '], shows that the branes have to satisfy a Dirac-type flux quantisation condition [1] $\left.\bar{z}_{1}\right]$. This leaves only a finite number of $\mathrm{SU}(2)$ conjugacy classes (for fixed gluing conditions), much in agreement with the CFT analysis. The remaining conjugacy classes are those where the angle $\theta$ introduced above takes the values $\theta_{n}=\frac{n}{\mathrm{k}} \pi$ for $n=0, \ldots, \mathrm{k}$. A different explanation for the discreteness of $\mathrm{SU}(2)$ branes was given in [2]1], where the stability of spherical branes was investigated with the help of the Born-Infeld action. It was found that only branes at 'latitudes' $\theta_{n}$ are stable against radial fluctuations; see also [222']

\section{Low-energy effective action}

After this account of the classical picture of branes in $\mathrm{SU}(2)$, let us now exploit the exact CFT description, the data of which were recalled in passing in section 2. Our aim is to calculate the low-energy effective action of open strings attached to such a brane. From this we can expect to gain new insight into the dynamics of branes wrapped on an $S^{2} \subset S^{3}$, but we can also hope to 'recognise' the resulting action as one naturally associated to a non-commutative space, which we might then refer to as the 'quantised' brane world-volume as felt by open strings. That deviations from the classical picture occur is suggested by analogy to flat branes and recalling that 2-branes in $\mathrm{SU}(2)$ carry a non-vanishing B-field.

Similar to the Seiberg-Witten analysis, we will concentrate on a certain regime of parameters, namely the low-energy limit $\alpha^{\prime} \rightarrow 0$ for large level k such that $\alpha^{\prime} \mathrm{k} \rightarrow \infty$, and with metric $G^{a b}$ and structure constants $f^{a b}$ in the following OPE of (rescaled) currents held fixed:

$$
\begin{aligned}
j^{a}\left(x_{1}\right) j^{b}\left(x_{2}\right)=\frac{\alpha^{\prime}}{2} & \frac{G^{a b}}{\left(x_{1}-x_{2}\right)^{2}} \\
& +\frac{i \alpha^{\prime} f^{a b}{ }_{c}}{x_{1}-x_{2}} j^{c}\left(x_{2}\right)+\ldots
\end{aligned}
$$

The metric $G$ is used in the following to raise and lower indices $a=1,2,3$. We concentrate on branes given by Cardy type boundary conditions. In the large $\mathrm{k}$ regime, there are vertex operators $V\left[\psi_{i}^{I}\right](x)$ present for $I=0,1, \ldots, 2 \alpha$. To these, we can associate effective fields $\mathrm{A} \in \operatorname{Mat}(2 \alpha+1)$ via $V[\mathrm{~A}]=\sum a_{I i} V\left[\psi_{i}^{I}\right]$. We will include stacks of $N$ identical branes from the start; this merely requires to replace the field $A$ from above by $A \in$ $\operatorname{Mat}(N) \otimes \operatorname{Mat}(2 \alpha+1)$. 
The boundary fields : $j^{a} V\left[\psi_{i}^{I}\right]:(x)$ have string masses given by

$$
M^{2}=\frac{1}{\alpha^{\prime}} h_{I}=\frac{I(I+1)}{\alpha^{\prime}(\mathrm{k}+2)}
$$

and become massless in the limit we consider. The combination $\Psi=: j^{a} V\left[\mathrm{~A}_{a}\right]$ : (with summation over $a$ understood) is physical if $\mathrm{L}^{a} \mathrm{~A}_{a}=0$, where the 'angular momentum' $\mathrm{L}_{a}$ acts by a matrix commutator,

$$
\mathrm{L}_{a} \mathrm{~A}=\left[\mathrm{Y}_{a}, * \mathrm{~A}\right]
$$

with $\mathrm{Y}_{a}=\mathbf{1}_{N} \otimes\left(1 / \sqrt{2 \alpha^{\prime}}\right) \mathrm{Y}_{a}^{1}$ denoting (rescaled) $\mathrm{su}(2)$-generators in $2 \alpha+1$ dimensions.

The mass squares above are eigenvalues of the operator $\mathrm{L}^{a} \mathrm{~L}_{a}$, and they induce a term quadratic in $\mathrm{L}_{a} \mathrm{~A}_{b}$ into the low-energy effective action. To obtain the higher order contributions, one has to compute $n$-point functions of the boundary field $\Psi$ (and then to integrate out world-sheet moduli), using the current OPE (5) as well as the following basic OPE:

$$
j^{a}\left(x_{1}\right) V[\mathrm{~A}]\left(x_{2}\right)=\frac{\alpha^{\prime} G^{a b}}{x_{1}-x_{2}} V\left[\mathrm{~L}_{b} \mathrm{~A}\right]\left(x_{2}\right)+\ldots
$$

The last piece of CFT information needed is that the OPE of lowest-dimension boundary fields $V\left[\psi_{i}^{I}\right]$ converges to a matrix product $*$ in the limit $\mathrm{k} \rightarrow$ $\infty$; this accounts for the matrix traces showing up later.

Details of the computation of the effective action (up to including fourth order terms in $A_{a}$ )

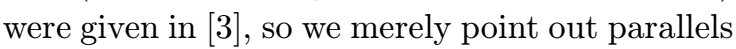
and differences to the case of flat branes here: In the OPEs $(5.15)$ and $(5.4)$, the $G^{a b}$ - and $\mathrm{L}^{a}$-terms on the rhs are familiar from the abelian situation, while the possibility to contract two currents into a third one, accompanied by a structure constant, will produce new terms. Our effective action should, however, reduce to the one for flat branes upon setting $\mathrm{f}_{a b c}=0$ and replacing $\mathrm{L}_{a}$ by $-i \partial_{a}$.

The result found in [3] does indeed have this property: It is suggestive to split the action

$$
\mathcal{S}_{\mathrm{eff}}=\mathcal{S}_{\mathrm{YM}}+\mathcal{S}_{\mathrm{CS}}
$$

into two pieces, with obvious nomenclature: Regarding $\mathrm{A}_{a}$ as a gauge field, we first define an associated field strength by

$$
\mathrm{F}_{a b}(\mathrm{~A})=i \mathrm{~L}_{a} \mathrm{~A}_{b}-i \mathrm{~L}_{b} \mathrm{~A}_{a}+i\left[\mathrm{~A}_{a}, \mathrm{~A}_{b}\right]+\mathrm{f}_{a b c} \mathrm{~A}^{c} ;
$$

then we have the usual expression for the 'YangMills part'

$$
\mathcal{S}_{\mathrm{YM}}=\frac{1}{4} \operatorname{tr}\left(\mathrm{F}_{a b} * \mathrm{~F}^{a b}\right) .
$$

Similarly, a 'Chern-Simons three-form' is introduced as

$$
\begin{aligned}
\mathrm{CS}_{a b c}(\mathrm{~A})= & \mathrm{L}_{a} \mathrm{~A}_{b} * \mathrm{~A}_{c} \\
& +\frac{1}{3} \mathrm{~A}_{a} *\left[\mathrm{~A}_{b} * \mathrm{~A}_{c}\right]-\frac{i}{2} \mathrm{f}_{a b d} \mathrm{~A}^{d} * \mathrm{~A}_{c},
\end{aligned}
$$

and the second part of our effective action reads

$$
\mathcal{S}_{\mathrm{CS}}=-\frac{i}{2} \operatorname{tr}\left(\mathrm{f}^{a b c} \mathrm{CS}_{a b c}\right)
$$

Note that both (15.6) and (15.81) include terms proportional to $\mathrm{f}_{a b c}$ which 'correct' the expressions familiar from ordinary geometry.

We should remark that, as usual in string theory, (15.5) follows from CFT only up to second order terms in $A_{a}$ that vanish on-shell, i.e. upon using the physical state condition $\mathrm{L}^{a} \mathrm{~A}_{a}=0$. The main motivation for this modification is that it renders the action off-shell gauge invariant under

$$
\delta_{\Lambda} \mathrm{A}_{a}=i \mathrm{~L}_{a} \Lambda+i\left[\mathrm{~A}_{a}, \Lambda\right]
$$

for arbitrary $\Lambda \in \operatorname{Mat}(N) \otimes \operatorname{Mat}(2 \alpha+1)$. Note, in particular, that the 'mass term' in the ChernSimons form (5.8) is required by gauge invariance. On the other hand, the effective action $(5.5)$ is the unique combination of $\mathcal{S}_{\mathrm{YM}}$ and $\mathcal{S}_{\mathrm{CS}}$ in which mass terms cancel - thus allowing for massless 'translation' modes.

In the case of flat branes in a B-field, the LEEA of open strings (in the Seiberg-Witten limit) could be recognised as a Maxwell theory on a deformed world-volume. In the present case, we expect quantisations of two-spheres to play a role, with

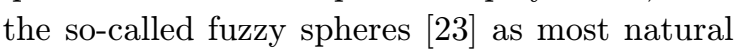
candidates. And indeed, the action $\mathcal{S}_{\mathrm{YM}}$ was already considered in the non-commutative geometry literature $\left[2 \overline{2} \overline{4}_{n}^{1}, \overline{2} \overline{2} \overline{2}\right]$, where it was derived from a Connes spectral triple and viewed as describing electrodynamics on a fuzzy sphere. In ['흠] , also Chern-Simons-like actions on such spaces were 
used. But before we pursue these non-commutative geometry ideas further, let us add some remarks on effective actions for full superstring theory backgrounds containing an SU(2) WZWmodel, and on what can be learnt from $\mathcal{S}_{\text {eff }}$ about the dynamics of $\mathrm{SU}(2)$-branes.

For string theory, we should extend all our calculations to the $N=1$ supersymmetric variant of the $\mathrm{SU}(2)$-model. But it was explained in [3. that the bosonic part of the supersymmetric effective action is again given by $\mathcal{S}_{\text {eff }}$ from eq. (5.5), at least in the limit $\alpha^{\prime} \rightarrow 0$ and $\alpha^{\prime} \mathrm{k} \rightarrow$ $\infty$. Moreover, it is this limiting regime and the fact that we focus on genus zero world-sheets which allows us to work in a space-time $\mathbb{R}^{7} \times S^{3}$; else we would have to deal with non-compact curved backgrounds like $\mathrm{AdS}_{3}$ or the linear dilaton which are part of the full superstring backgrounds listed before. Boundary conditions for such models are hard to construct, although significant progress for the boundary Liouville model has been reported on at this conference [ī

The action (5.5) can be viewed as a potential for 'Higgs fields' associated with internal degrees of freedom of branes wrapped around (fuzzy) spheres. The study of its equations of motion should provide interesting clues e.g. on stability and bound states.

Owing to the (non-commutative) geometric nature of $\mathcal{S}_{\mathrm{YM}}$ and $\mathcal{S}_{\mathrm{CS}}$, the equations of motion derived from $\mathcal{S}_{\text {eff }}$ take the rather simple form

$$
\mathrm{L}^{a} \mathrm{~F}_{a b}+\left[\mathrm{A}^{a}{ }^{*} \mathrm{~F}_{a b}\right]=0 \quad:
$$

The curvature of the solutions must be covariantly constant.

As indicated above, and as suggested by (classical) target symmetry, rigid rotations of the initial stack of $N$ branes of type $\alpha$ correspond to solutions of ( $(\overline{5} \cdot \overline{10})$, more precisely to 'constant' solutions - ones with $\mathrm{L}_{a} \mathrm{~A}_{b}=0$ for all $a, b$ - which moreover satisfy $\left[\mathrm{A}_{a}, \mathrm{~A}_{b}\right]=0$. These are familiar from the flat case $\left[\overline{2} \overline{8}_{1}^{\prime}\right]$

On 'constant' gauge configurations, eqs. ('is.ioj) simplify to

$$
\left[\mathrm{A}^{a},\left[\mathrm{~A}_{a}, \mathrm{~A}_{b}\right]-i \mathrm{f}_{a b c} \mathrm{~A}^{c}\right]=0,
$$

from which another class of solutions become obvious: constant gauge fields which moreover sat- isfy

$$
\left[\mathrm{A}_{a}, \mathrm{~A}_{b}\right]=i \mathrm{f}_{a b}{ }^{c} \mathrm{~A}_{c}
$$

i.e. which form generators of a (not necessarily irreducible) representation of $\mathrm{su}(2)$ by matrices in $\operatorname{Mat}(N) \otimes \mathbf{1}_{2 \alpha+1}$ (the requirement $\mathrm{L}_{a} \mathrm{~A}_{b}=0$ fixes the second tensor factor of $A_{a}$ to be trivial). Putting $\mathrm{f}_{a b c}=0$, these coincide with the rigid rotations, but they have quite different effects in the non-abelian case, as we will point out shortly. Written in terms of new variables $\mathrm{B}_{a}:=\mathrm{Y}_{a}+$

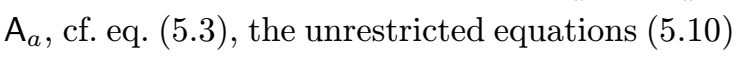
acquire the same form

$$
\left[\mathrm{B}^{a},\left[\mathrm{~B}_{a}^{*}, \mathrm{~B}_{b}\right]-i \mathrm{f}_{a b c} \mathrm{~B}^{c}\right]=0
$$

as those in $(\overline{5} \cdot \overline{1} \overline{1})$. This means that any su(2)-representation $\overline{\mathrm{B}}_{a}^{-} \in \operatorname{Mat}(N) \otimes \operatorname{Mat}(2 \alpha+1)$ solves the equations of motion.

According to standard string theory reasoning, each stationary point $\mathrm{A}_{a}$ of $\mathcal{S}_{\text {eff }}$ should correspond to a zero of the $\beta$-function that describes perturbations by our world-sheet field $\Psi$. At such a fixed point of the associated RG-flow, conformal invariance is restored and, in particular, a conformal boundary condition is reached. The new boundary condition, resp. the brane system corresponding to it, can be characterised with the help of world-sheet methods: For the simple constant commuting case from above, this is straightforward since $\Psi$ is a marginal boundary operator, so one can use the results of [i20 i]. All the other solutions to the equations of motion belong to RG flows induced by perturbations

$$
S_{\text {pert }}=\int d x: j^{a} V\left[\mathrm{~A}_{a}\right]:(x)
$$

with non-marginal (or 'marginally relevant') fields which destroy conformal invariance.

In order to pin down the boundary conditions at the RG fixed points corresponding to $\mathrm{su}(2)$ representations, one can first make an educated guess resting on symmetry considerations, and then test this conjecture with techniques from conformal perturbation theory, see [i] and The observations on the (world-sheet) symmetry of the new boundary conditions are most easily understood in the case of constant solutions $(5 . \overline{12})$, where the perturbing field is given by the product of a boundary current $j^{a}$ with a constant 
$\mathrm{SU}(2)$ boundary spin $\mathrm{A}_{a}$. Because of (5.12), the operators $\operatorname{ad}\left(\mathrm{A}_{a}\right)$ furnish an $\mathrm{su}(2)$-representation on $\operatorname{Mat}(N)$. Turning on the perturbation $j^{a} \mathrm{~A}_{a}$ breaks the individual su(2) resp. $\widehat{\mathrm{SU}}(2)_{\mathrm{k}}$ symmetry generated by $a d\left(\mathrm{~A}_{a}\right)$ resp. $j^{a}$, but from experience with similar couplings of spin and orbital angular momentum in quantum mechanics, one may suspect that the sum $j^{a}+a d\left(\mathrm{~A}^{a}\right)$ has a good chance to be realized as a symmetry in the perturbed theory. The relations of $j^{a}+a d\left(\mathrm{~A}^{a}\right)$ are indeed those of an $\widehat{\mathrm{SU}}(2)_{\mathrm{k}}$ current algebra, acting on $\operatorname{Mat}(N) \otimes \mathcal{H}_{\alpha}$, where $\mathcal{H}_{\alpha}$ is the state space of a single brane with label $\alpha$. If we assume that this action survives the perturbation, we may conclude that the new brane configuration is built up from Cardy branes (here the rationality of the WZW model is important). A little more thought and guesswork leads to the conjecture that the fixed point corresponding to a solution of ( $(5.10 i)$ of the type $\left[\mathrm{B}_{a}, \mathrm{~B}_{b}\right]=i \mathrm{f}_{a b}{ }^{c} \mathrm{~B}_{c}$ is determined by the representation content of the matrices $\mathrm{B}_{a} \in \operatorname{Mat}(N) \otimes \operatorname{Mat}(2 \alpha+1)$; we have passed to non-constant solutions here since the pattern is no more difficult to describe for them:

Denote by $\left(n_{i}\right)_{i=1, \ldots, r}$ the partition $n_{1}+\ldots+n_{r}=$ $(2 \alpha+1) N$ where the $n_{i}$ are dimensions of $\operatorname{su}(2)$ irreducibles contained in the representation given by $\mathrm{B}_{a}$. Then, under the perturbation by $j^{a} \mathrm{~A}_{a}$, the stack of $N$ branes of type $\alpha$ evolves into a system that contains $N_{\beta}$ Cardy branes of type $\beta$, where $N_{\beta}=\#\left\{n_{i} \mid n_{i}=2 \beta+1\right\}$.

It is, in fact, not too difficult to give quantitative support for this conjecture, by computing the socalled $g$-factor or boundary entropy [ $\left[2 \bar{g}_{1}^{\prime}\right]$ which is simply the 1-point function of the identity operator. For a single brane of type $\alpha$ in the $\mathrm{SU}(2)$ WZW model, it is given by [i

$$
g_{\alpha}=\frac{S_{\alpha 0}}{\sqrt{S_{00}}}=\left(\frac{2}{\mathrm{k}+2}\right)^{\frac{1}{4}} \frac{\sin \frac{(2 \alpha+1) \pi}{\mathrm{k}+2}}{\sin ^{\frac{1}{2}} \frac{\pi}{\mathrm{k}+2}},
$$

and it behaves additively under forming superpositions (systems) of branes. But this means that the set of all $g$-factors for superpositions of Cardy branes in $\mathrm{SU}(2)$ form a lattice - and any member can be identified by computing the first few terms of a perturbative expansion (here: in $1 / k$ ) of $g$ at the new fixed point, in complete analogy to the discussion of boundary perturbations in
Virasoro minimal models [4i] logarithm of the 'vacuum functional' $g$ in such an expansion is nothing but computing low-order contributions to the effective action, the result of which was presented already above. And indeed one finds that

$$
\mathcal{S}_{\text {eff }}\left(\mathrm{A}_{a}\right)=\log \frac{g_{\mathrm{A}}}{g_{N \cdot \alpha}},
$$

where $g_{N \cdot \alpha}=N g_{\alpha}$ denotes the boundary entropy of the original $N$ branes of type $\alpha$, while $g_{\mathrm{A}}$ denotes that of the end-configuration corresponding to the solution $\mathrm{A}_{a}$, according to the rule stated above.

As a special case of such an RG flow, consider $N$ branes of type $\alpha=0$ and let $\mathrm{A}_{a} \in \operatorname{Mat}(N)$ generate the irreducible $\mathrm{su}(2)$-representation in the $N$-dimensional Chan-Paton space. Then the rule implies that the system flows to a single brane of type $\beta=\frac{N-1}{2}$. Comparison of the actions evaluated at both configurations shows that energy is released during that flow, i.e. that in the $\mathrm{SU}(2)$ WZW models, we can indeed regard ('spherical') Cardy branes of type $\alpha>0$ as bound states of $2 \alpha+1$ zero-branes.

It would be interesting to see whether similar statements hold in other examples of CFT string compactifications. It should be possible to treat e.g. Gepner models by the same methods. At least for B-type branes, this could lead to quantisations of supersymmetric cycles (here of holomorphic submanifolds of the Calabi-Yau manifold in question). In contrast, one does not expect that the world-volume of an A-type brane is deformed by open string effects, at least if one relies on the large-volume limit and the flat case as guidelines. Then, it follows from the Atype supersymmetric cycle conditions that the brane carries no B-field. Note that this might imply that ordinary submanifolds can be mirrors of quantised ones.

\section{Towards spectral data for branes}

The $\mathrm{SU}(2)$ example shows that we can derive brane effective actions, and sometimes interesting dynamical effects encoded in them, purely from boundary CFT without ever invoking geo- 
metrical concepts, be they classical or non-commutative. From this point of view, it comes as no surprise that actions describing field theories 'on a brane world-volume' are sometimes (or rather: usually) not of a type known from classical differential geometry. The remarkable fact is rather that, at least in the examples studied so far, these actions can still be connected with natural functionals on non-commutative spaces, which one may in hindsight identify with brane worldvolumes. This fact makes it tempting to leave the secure path of world-sheet computations and replace (or at least supplement) it by geometrical target space considerations, although now in the much wider sense of Connes' non-commutative geometry.

What we have in mind is a reformulation of the 'recipe' commonly used in string theory when one is to derive low-energy effective actions: Instead of going through the sometimes extremely tedious CFT calculations step by step, it is often enough to appeal to certain 'principles' - concerning (super)symmetries, e.g. - to be able to simply write down the action in question. These principles in particular include the conviction that low-energy effective actions induced by string theory are of a geometrical nature. It is hard to see how the validity of such a principle could be derived in any generality from, say, the CFT formulation of perturbative strings, and the existence of 'non-commutative actions' for certain branes shows that the classical setting is definitely too narrow. But since this 'principle of being geometric' has proven so useful, one should try to widen it appropriately so as to include the geometry of quantised spaces.

The main difficulties are encountered with the zeroth step - the one not to worry about in the classical situation: There, it is more or less evident which target space one is talking about, and all the work consists in finding appropriate actions on it. The lesson to be learnt from branes is not to presuppose too much about the nature of the brane world-volume itself, but rather to carefully uncover it from an 'unbiased' but ungeometric description of the brane as provided by boundary CFT.

Already in order to state the problem more precisely, we need to recall how spaces are presented in non-commutative geometry. Instead of local charts, one uses so-called spectral data including an algebra $\mathcal{A}$, a (generalised) 'Dirac operator' $D$ and a Hilbert space $\mathcal{H}$ on which $\mathcal{A}$ and $D$ act. The actions must meet various conditions, in particular ones concerning boundedness properties. Here, we restrict ourselves to a very crude list of ingredients and refer to Connes' book [30'] or also to ["5].] for the details.

The idea to use data $(\mathcal{A}, \mathcal{H}, D)$ to formulate geometry is of course inspired by quantum mechanics: An electron moving on a (spin) manifold $M$ 'sees' an algebra $\mathcal{A}=C(M)$ or $C^{\infty}(M)$ of functions on $M$ generated by position operators, along with a space of states $\mathcal{H}=L^{2}(S)$ - squareintegrable sections of the spinor bundle - and the Dirac operator. Roughly speaking, the algebra $\mathcal{A}$ encodes the topology of the space under consideration (in the commutative case, this is just Gelfands theorem), while the choice of a ' $K$-cycle' $(\mathcal{H}, D)$ fixes the geometry. That this can be made to work quantitatively is of course a matter of proving mathematical theorems "i] $3 \overline{0}$. In the process, one has to give algebraic reformulations (and generalisations) of all the basic notions from classical geometry, including in particular differential forms: Given $(\mathcal{A}, \mathcal{H}, D)$, the space of 1 -forms is defined as

$$
\Omega_{D}^{1}(\mathcal{A})=\left\{\sum_{i, j} \pi\left(a_{i}\right)\left[D, \pi\left(a_{j}\right)\right] \mid a_{i}, a_{j} \in \mathcal{A}\right\}
$$

where $\pi$ is the representation of $\mathcal{A}$ on $\mathcal{H}$. In particular, forms are linear operators on the Hilbert space, and the definition works irrespective of whether the elements of $\mathcal{A}$ commute with each other or not. We refrain from repeating the definition of higher forms since this involves some technical subtleties, see $[\overline{30}, \overline{1}, \overline{5}, \overline{1}]$; for the present purposes, it is sufficient to know the differential of a 1 -form $A$ as given explicitly in (6.1):

$$
d A=\sum_{i, j}\left[D, \pi\left(a_{j}\right)\right]\left[D, \pi\left(a_{j}\right)\right] .
$$

Suppose the non-commutative space is additionally equipped with an integral over differential forms', i.e. a linear map $f: \Omega_{D}^{\bullet}(\mathcal{A}) \longrightarrow \mathbb{C}$ with the cyclicity property $f \eta \omega=f \omega \eta$. Then we can already formulate an action for 'electrodynamics' on this non-commutative space: Re- 
gard $A$ as a 1 -form gauge potential and define the corresponding field strength 2 -form by $F=$ $d A+A A$. Then

$$
\mathcal{S}_{\mathrm{Maxw}}=f F^{2}
$$

is invariant under the gauge transformations $A \mapsto$ $u A u^{*}+u d u *$ for any unitary $u \in \mathcal{A}$, as can be seen with the help of the (graded) Leibniz rule for $d$ and cyclicity of the integral. Of course, $\mathcal{S}_{\text {Maxw }}$ is but the simplest example of a 'geometric' action that can be associated with a spectral triple. Using algebraic generalisations of other standard tools of geometry (e.g. of vector bundles, which appear as projective $\mathcal{A}$-modules), one can write down more complicated functionals, but as in classical geometry one expects that a few preferred actions remain when exploiting naturality and symmetry principles.

Before we try to link up the general non-commutative geometry framework to branes, let us add some remarks on possible variants. First of all, it should be said that the algebraic reformulation of notions from classical geometry is typically not unique. Any such reformulation must meet the requirement that ordinary geometry is reproduced in the commutative case, but often there are different generalisations which happen to coincide when $\mathcal{A}$ is commutative. Choices between different definitions can be made on the grounds of mathematical power, or of physical intuition. Secondly, and of more practical consequences for applications in string theory, one can work with variations of Connes' spectral triple. Let us again try to motivate this by looking at a quantum mechanical particle moving on a manifold. When dealing with a spin-less boson, neither spinor bundle nor Dirac operator are available, but one can still extract geometrical information on the space from the spectral data $(\mathcal{A}, \mathcal{H}, \square)$ where $\mathcal{H}=L^{2}\left(\Lambda^{\bullet} M\right)$ and where $\square$ denotes the Laplace operator [i,i]. On the other hand, if positronium moves on a manifold, two operators are available (two Dirac operators or, alternatively, the differential and its adjoint) from which it is in fact easier to reconstruct differential geometry than from a single Dirac operator. This is explained in great detail in [ it was also shown how classes of classical geome- tries (e.g. Riemann, Kähler, Hyperkähler) correspond to a hierarchy of algebraic relations among a set of generalised Dirac operators, which can also be seen as a hierarchy of supersymmetries acting on these spectral data.

Now let us try to devise a procedure by which we assign a set of spectral data to a brane given by a conformal boundary condition. The recipe to be given remains tentative, should be tested in more examples and may need revision in certain places. It is motivated by the calculations that lead to non-commutative effective actions as reviewed above, and equally strongly by the ideas

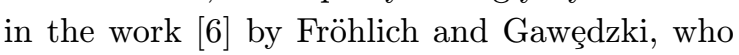
were the first to try and extract (in the closed string case) non-commutative spaces from CFT. The main piece of data we have to recover is the algebra $\mathcal{A}$ of 'functions' on the world-volume of the brane. In the two examples studied above, these 'functions' contain the effective fields appearing in the effective brane action. These are in turn obtained from the open string vertex operators which are supported by the boundary condition in question, and multiplication of effective fields is inherited from the OPE of the corresponding boundary fields. However, only a subset of all boundary fields appeared in the Maxwell actions on the non-commutative plane or on a fuzzy sphere. This was due to the fact that we considered only a specific regime of parameter space, including the limit $\alpha^{\prime} \rightarrow 0$, so that all higher excitations (non-lowest dimension fields) become infinitely massive and disappear. Accordingly, the following proposal for the definition of the world-volume algebra $\mathcal{A}_{\alpha}$ of a brane $\alpha$ begins by choosing a subspace $\mathcal{V}$ of all boundary fields (or, equivalently, of the state space $\mathcal{H}_{\alpha}$ of the boundary CFT). Typically, $\mathcal{V}$ will precisely contain the 'survivors' of a Seiberg-Witten-like limit. This limit is then also to be applied to all structure constants in the theory. To each $\psi_{i} \in \mathcal{V}$, we associated a (linear) generator $\hat{\psi}_{i}$ of $\mathcal{A}_{\alpha}$. Multiplication is defined via the OPE:

$$
\hat{\psi}_{i} * \hat{\psi}_{j}:=\pi_{\mathcal{V}}\left(\psi_{i}(1) \psi_{j}(0)\right)^{\wedge}
$$

where $\pi_{\mathcal{V}}$ denotes orthogonal projection along $\mathcal{V}$ of the operators appearing in the expansion on the rhs, and is again meant to include performing 
the limits involved also for the OPE structure constants.

Insertion points 1 and 0 in $(\underline{6} \cdot \overline{2} \overline{1})$ are chosen to remove world-sheet coordinates from the rhs. In the two examples encountered above, such an arbitrary choice is not necessary since the SWlimits taken there, together with the truncations to $\mathcal{V}$, remove all the coordinate dependence from $\pi_{\mathcal{V}}\left(\psi_{i}\left(x_{1}\right) \psi_{j}\left(x_{2}\right)\right)$ - simply because the limits imply that the conformal dimensions (scaled appropriately with $\alpha^{\prime}$ ) of the open string vertex operators in $\mathcal{V}$ vanish.

Assume that the limiting regime under consideration is such that the whole boundary CFT becomes topological in the sense that the correlation functions are coordinate independent. Then it is rather easy to prove associativity of the product defined by the OPE: The coefficients in the multiplication table can be visualised by disk diagrams with three insertion of boundary fields, associativity requires to look at 4-point functions. But as long as fields can be moved close to one another or further apart without changing the value of the diagram, associativity of $*$ follows directly from deforming the insertion points. The same reasoning would of course apply to topological closed strings, replacing disks with spheres and open with closed string vertex operators. In this case, however, one can even move the corresponding bulk fields around each other, so that, on a topological level, no non-commutative effects should appear for closed strings. This is to be contrasted to the open string case, where even from the crude point of view of world-sheet topology there is no reason to expect that a diagram $\left\langle\psi_{i} \psi_{j} \psi_{k}\right\rangle$ equals $\left\langle\psi_{j} \psi_{i} \psi_{k}\right\rangle$ : The mutual non-locality of boundary operators may survive even in a 'topological' limit, as is the case for flat branes in a B-field or for the $\mathrm{k} \rightarrow \infty$ limit of $\mathrm{SU}(2)$ branes.

Clearly, not every limiting/projection scheme $\mathcal{V}$ yields an associative product $*$. SU(2) provides interesting examples where one can trace back violations of associativity to non-vanishing of the 3form field strength $H$, see [2] [n all cases encountered so far, any non-associativity introduced by the projection/limiting procedure amounts to a relatively mild form of quasi-associativity, because of the properties of the CFT and its Moore-
Seiberg category. One should note that there is a slightly different definition of a world-volume algebra that avoids non-associativity from the start [";]]: One first chooses a Hilbert space $\mathcal{H}$, then defines the algebra in terms of matrix elements of linear operators acting in $\mathcal{H}$. In principle, however, non-associativity might be of similar significance as non-commutativity, even though it is hard to fit into existing frameworks of noncommutative geometry.

But let us return to our concrete examples and see which world-volume algebras arise from applying the general recipe there; all the necessary CFT data were provided before. For a flat brane in a constant B-field, considered in the SeibergWitten limit, the world-volume generators are the $\mathrm{U}(1)^{2}$ primaries $\exp \{i \vec{k} \cdot \vec{X}\}$; all descendants are discarded since they become infinitely much heavier as $\alpha^{\prime} \rightarrow 0$. In this limit, the OPE of two such fields becomes

$$
e^{i \vec{k}_{1} \cdot \vec{X}}\left(x_{1}\right) e^{i \vec{k}_{2} \cdot \vec{X}}\left(x_{2}\right)=e^{i \pi \vec{k}_{1}^{\mathrm{t}} \Theta \vec{k}_{2}} e^{i\left(\vec{k}_{1}+\vec{k}_{2}\right) \cdot \vec{X}}\left(x_{2}\right)
$$

after projecting out descendants. It is insertion point independent and coincides with the formula for the Moyal product of exponentials. We thus arrive at the (associative) algebra of a deformed plane, without detour through an effective action.

For the SU(2) WZW model, we chose to consider branes in the combined limit $\alpha^{\prime} \rightarrow 0$ and $\alpha^{\prime} \mathrm{k} \rightarrow \infty$. Then, only the SU(2)-primaries along with their $j_{0}^{a}$-descendants retain finite string theory mass and provide $(2 \alpha+1)^{2}$ linear generators $\psi_{i}^{I}$ of the world-volume algebra, where again $\alpha$ is the label of the Cardy brane. We have mentioned before that the (truncated) OPE of $\psi_{i}^{I}$ becomes $x_{i}$-independent in the limit, and that the structure constants become those of ordinary matrix multiplication. More precisely, one can view $\operatorname{Mat}(2 \alpha+1)$ as a module of $\operatorname{su}(2)$, acting by commutators with the generators in $2 \alpha+1$ dimensions, and decompose it into irreducibles,

$$
\operatorname{Mat}(2 \alpha+1) \simeq_{\mathrm{su}(2)} \bigoplus_{J=0,1, \ldots, 2 \alpha} V^{J} .
$$

One first notes that the same $\mathrm{su}(2)$ multiplets as in the (lowest energy subspaces of) $\mathcal{H}_{\alpha}$ show up. Next, one can compare the (limit of the) 
boundary OPE structure constants for the $\psi_{i}^{I}$ with the multiplication that is induced on the rhs of $(\underline{6} . \overline{3})$ by means of the isomorphism to the algebra on the lhs [32i]. One finds that both multiplication tables agree. On the other, this isomorphism is nothing but an elegant way to define an associative truncation of the algebra of functions on a two-sphere, yielding a fuzzy sphere. Again, we arrive at the non-commutative space that fits with the effective action computed independently.

Having found an algebra of 'functions on the brane world-volume', one should still pick a $K$-cycle $(\mathcal{H}, D)$ to determine the geometrical structure of the (non-commutative) space under consideration. Proposals how to obtain these data from a CFT were made in [ín] for the closed string case: As a Hilbert space, one simply takes a suitable subspace of the CFT state space - e.g. in the supersymmetric situation, the zero momentum Ramond states are a natural choice. Candidates for non-commutative generalisations of Laplace or Dirac operators are provided by generators of the (super) Virasoro algebra: One puts $\square=L_{0}-c / 24$ or $D=G_{0}$, restricted to the Hilbert space $\mathcal{H}$ chosen.

One should now 'test' these proposals in the open string context, i.e. derive differential forms and 'geometric' actions for such spectral data and compare them to the effective actions computed with world-sheet methods. For the case of flat branes, there is little doubt that the effective action (15.5) will be recovered, since $L_{0}-c / 24$ resp. $G_{0}$ simply coincide with the Laplacian resp. the Dirac operator on the (classical) world-volume, all the non-commutativity being contained in the *-product (see e.g. [i5, $3 \overline{1}_{i}^{\prime}$ for details). To derive the effective action (5.5) for $\mathrm{SU}(2)$ branes from spectral data recovered from string theory would provide a more severe test - which is left for future work.

\section{References}

[1] J. Fröhlich, The non-commutative geometry of two-dimensional supersymmetric conformal field theory, in: PASCOS, Proc. of the Fourth Intl. Symp. on Particles, Strings and Cosmology, K.C. Wali (ed.), World Scientific 1995
[2] A.Yu. Alekseev, A. Recknagel, V. Schomerus, Non-commutative world-volume geometries: Branes on $S U(2)$ and fuzzy spheres, J. High Energy Phys. 9909 (1999) 023, hepth/9908040

[3] A.Yu. Alekseev, A. Recknagel, V. Schomerus, Brane dynamics in background fluxes and noncommutative geometry, J. High Energy Phys. 0005 (2000) 010, hep-th/0003187

[4] A. Recknagel, D. Roggenkamp, V. Schomerus, On relevant boundary perturbations in unitary minimal models, hep-th/0003110

[5] J. Fröhlich, O. Grandjean, A. Recknagel, Supersymmetric quantum theory, non-commutative geometry, and gravitation, Les Houches Lecture Notes 1995, hep-th/9706132; Supersymmetric quantum theory and differential geometry, Commun. Math. Phys. 193 (1998) 527, hepth/9612205; Supersymmetric quantum theory and non-commutative geometry, Commun. Math. Phys. 203 (1999) 119, math-ph/9807006

[6] J. Fröhlich, K. Gawędzki, Conformal field theory and the geometry of strings, CRM Proceedings and Lecture Notes Vol. 7 (1994) 57, hepth/9310187

[7] A. Recknagel, V. Schomerus, D-branes in Gepner models, Nucl. Phys. B531 (1998) 185, hepth/9712186

[8] J.L. Cardy, Boundary conditions, fusion rules and the Verlinde formula, Nucl. Phys. B324 (1989) 581

[9] D.C. Lewellen, Sewing constraints for conformal field theories on surfaces with boundaries, Nucl. Phys. B372 (1992) 654

[10] I. Runkel, Boundary structure constants for the A-series Virasoro minimal models, Nucl. Phys. B549 (1999) 563, hep-th/9811178

[11] R.E. Behrend, P.A. Pearce, V.B. Petkova, J.-B. Zuber, Boundary conditions in rational conformal field theories, hep-th/9908036

[12] G. Felder, J. Fröhlich, J. Fuchs, C. Schweigert, Conformal boundary conditions and threedimensional topological field theory, Phys. Rev. Lett. 84 (2000) 1659, hep-th/9909140; Correlation functions and boundary conditions in RCFT and three-dimensional topology, hepth/9912239

[13] V. Schomerus, D-branes and deformation quantization, J. High Energy Phys. 9906 (1999) 030, hep-th/9903205 
[14] N. Seiberg, E. Witten, String theory and noncommutative geometry, J. High Energy Phys. 9909 (1999) 032, hep-th/9908142

[15] M.R. Douglas, C. Hull, D-branes and the noncommutative torus, J. High Energy Phys. 9802 (1998) 008, hep-th/9711165

[16] C.G. Callan, J.A. Harvey, A. Strominger, World sheet approach to heterotic instantons and solitons, Nucl. Phys. B359 (1991) 611; Supersymmetric string solitons, hep-th/9112030

[17] A.Yu. Alekseev, V. Schomerus, D-branes in the WZW model, Phys. Rev. D60 (1999) 061901, hep-th/9812193

[18] K. Gawędzki, Conformal field theory: a case study, hep-th/9904145

[19] S. Stanciu, D-branes in group manifolds, J. High Energy Phys. 0001 (2000) 025, hep-th/9909163

[20] A. Recknagel, V. Schomerus, Boundary deformation theory and moduli spaces of D-branes, Nucl. Phys. B545 (1999) 233, hep-th/9811237; Moduli spaces of D-branes in CFT-backgrounds, hep-th/9903139

[21] C. Bachas, M. Douglas, C. Schweigert, Flux stabilization of D-branes, J. High Energy Phys. 0005 (2000) 048, hep-th/0003037

[22] J. Pawelczyk, SU(2) WZW D-branes and their non-commutative geometry from DBI action, J. High Energy Phys. 0008 (2000) 006, hepth/0003057

[23] J. Madore, The fuzzy sphere, Class. Quant. Grav. 9 (1992) 69; Fuzzy space-time, grqc/9607065

[24] J. Madore, An Introduction to Noncommutative Differential Geometry and its Physical Applications, Cambridge University Press 1999

[25] U. Carow-Watamura, S. Watamura, Noncommutative geometry and gauge theory on fuzzy sphere, Commun. Math. Phys. 212 (2000) 395, hep-th/9801195

[26] C. Klimčík, A nonperturbative regularization of the supersymmetric Schwinger model, Commun. Math. Phys. 206 (1999) 567, hepth/9903112

[27] J. Teschner, Remarks on Liouville theory with boundary, hep-th/0009138

[28] E. Witten, Bound states of strings and Dbranes, Nucl. Phys. B460 (1996) 335, hepth/9510135
[29] I. Affleck, A.W.W. Ludwig, Exact conformal field theory results on the multichannel Kondo effect: Single-fermion Green's function, selfenergy, and resistivity, Phys. Rev. B48 (1993) 7297; Universal noninteger 'groundstate degeneracy' in critical quantum systems, Phys. Rev. Lett. 67 (1991) 161

[30] A. Connes, Noncommutative Geometry, Academic Press 1994

[31] O. Grandjean, Non-commutative differential geometry, Ph.D. Thesis, ETH Zürich, July 1997

[32] J. Hoppe, Diffeomorphism groups, quantization and $S U(\infty)$, Int. J. Mod. Phys. A4 (1989) 5235 